The Astrophysical Journal, 372:L75-L78, 1991 May 10

(C) 1991. The American Astronomical Society. All rights reserved. Printed in U.S.A.

\title{
CODED-APERTURE IMAGING OF THE GALACTIC CENTER REGION AT GAMMA-RAY ENERGIES
}

\author{
Walter R. Cook, John M. Grunsfeld, William A. Heindl, David M. Palmer, Thomas A. Prince, \\ STEPHEN M. Schindler, AND EDWARd C. STONE \\ Division of Physics, Mathematics, and Astronomy, California Institute of Technology, M.S. 220-47, Pasadena, CA 91125 \\ Received 1990 December 26; accepted 1991 February 26
}

\begin{abstract}
The first coded-aperture images of the Galactic center region at energies above $30 \mathrm{keV}$ have revealed two strong $\gamma$-ray sources. One source has been identified with the X-ray source 1E $1740.7-2942$, located 0.8 away from the nucleus. If this source is at the distance of the Galactic center, it is one of the most luminous objects in the galaxy at energies from 35 to $200 \mathrm{keV}$. The second source is consistent in location with the X-ray source GX 354+0 (MXB 1728-34). In addition, $\gamma$-ray flux from the location of GX $1+4$ was marginally detected at a level consistent with other post-1980 measurements. No significant hard X-ray or $\gamma$-ray flux was detected from the direction of the Galactic nucleus $\left(\operatorname{Sgr} \mathrm{A}^{*}\right)$, or from the direction of the recently discovered $\gamma$-ray source GRS $1758-258$.
\end{abstract}

Subject headings: galaxies: The Galaxy — gamma rays: general — X-rays: sources

\section{INTRODUCTION}

Observations from Spacelab 2 (Skinner et al. 1987) and Spartan 1 (Kawai et al. 1988) between 1 and $30 \mathrm{keV}$, together with earlier results from the Einstein observatory between 0.9 and $4 \mathrm{keV}$ (Watson et al. 1981; Hertz \& Grindlay 1984), identified several point sources within approximately $1^{\circ}$ of the Galactic nucleus, including a point source at the nucleus itself. At higher energies, observations by nonimaging instruments with wide fields of view (FWHM $>15^{\circ}$ ) detected $0.511 \mathrm{MeV}$ positron annihilation line radiation and hard continuum emission extending above $1 \mathrm{MeV}$ from the general direction of the Galactic center (e.g., Riegler et al. 1981; Leventhal et al. 1982; Riegler et al. 1985; Leventhal et al. 1989). Both line and continuum components were found to vary in intensity on time scales as short as 6 months. The compact source size required by the time variability, and the unusual $\gamma$-ray spectrum, have stimulated considerable speculation on the nature of the source of emission.

In this paper, we report $\gamma$-ray flux measurements based on the first coded-aperture imaging observations of the Galactic center region at $\gamma$-ray energies $(30 \mathrm{keV}-7 \mathrm{MeV})$. The initial report of these observations (Cook et al. 1989) discussed the detection of a strong $\gamma$-ray source that was tentatively identified as the X-ray source 1E 1740.7-2942 (Hertz \& Grindlay 1984), consistent with the Spacelab 2 observation of this source as dominant in the region at $19-30 \mathrm{keV}$ (Skinner et al. 1987). Further analysis of our data confirmed 1E 1740.7-2942 as the source of the primary $\gamma$-ray emission, and in addition revealed a second source, consistent in location with the $\mathrm{X}$-ray burst source GX 354+0 (Cook et al. 1990a). Here we present improved $\gamma$-ray flux measurements for 1E $1740.7-2942$ and GX $354+0$.

\section{OBSERVATIONS}

The observations were performed with the Caltech GammaRay Imaging Payload (GRIP), a balloon borne coded-aperture telescope sensitive to photons in the energy range from $30 \mathrm{keV}$ to $10 \mathrm{MeV}$ (Althouse et al. 1985). The instrument employs a rotating hexagonal-celled uniformly redundant array (HURA) and a position-sensitive $\mathrm{NaI}(\mathrm{Tl})$ scintillation detector to image a $14^{\circ}$ diameter field of view with 1.1 angular resolution.

The Galactic center region was observed for two $4 \mathrm{hr}$ periods during the interval 1988 April 12.62 to $13.00 \mathrm{UT}$, as part of a $30 \mathrm{hr}$ balloon flight of the instrument from Alice Springs, NT, Australia. The data were processed in seven segments of somewhat more than one hour each. For each segment a single strong point source of $\gamma$-ray emission was detected at energies between 35 and $200 \mathrm{keV}$. Data was available from an on-board CCD star camera to determine the absolute position of the source emission in four of the seven observation periods, leading to the identification of the strong $\gamma$-ray source as $1 \mathrm{E} 1740.7-2942$ (Cook et al. 1990a). The remaining three observation periods occurred during daylight when no stars were visible in the CCD camera.

We used the strong source, identified as $1 \mathrm{E} 1740.7-2942$, as a pointing reference to co-align images from all the separate 1 hr segments. This procedure reduced possible blurring effects resulting from pointing uncertainties and allowed images from all the segments, including the last three which lacked star camera data, to be combined in a search for weaker sources. A second source was detected in the energy range from 23 to 122 $\mathrm{keV}$, as shown in Figure 1 (where the primary peak has been aligned with 1E 1740.7-2942). The presence of the second peak, consistent in location with the known X-ray source, GX $354+0$, further supported the identification of the primary peak with 1E $1740.7-2942$.

Flux measurements and upper limits for 1E 1740.7-2942 and GX $354+0$, are presented in Table 1 and Figure 2. The flux values were computed as weighted averages of values obtained in each of the seven $1 \mathrm{hr}$ segments, with weights chosen to minimize the statistical error. The use of detailed instrument calibrations, including laboratory measurements of photon attenuation in passive instrument material, and the angular response of the lead collimator, has improved the accuracy of the present measurements, compared with those presented previously (Cook et al. 1988, 1990a). Flux measurements for the Crab Nebula and Pulsar, also made during the 1988 April flight, agree with previous measurements (Jung 1989 , and references therein) within approximately $10 \%$ at 


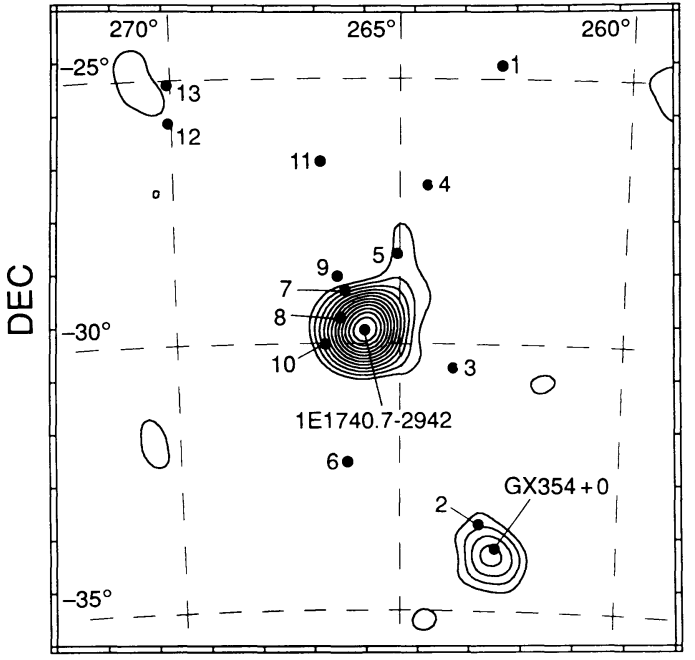

RA

FIG. 1.- Image of the Galactic center region from 23 to $122 \mathrm{keV}$. Right ascension (vertical lines) and declination (horizontal lines) are indicated for epoch 1988.3. The contours indicate the number of excess counts in a given direction, calibrated in units of the statistical significance of the excess, with contours beginning at the $2 \sigma$ level and spaced by $1 \sigma$. Sources $1 \mathrm{E}$ $1740.7-2942$ and GX $354+0$ are labeled by name, while other known hard X-ray sources (Bradt \& McClintock 1983; Levine et al. 1984; Skinner et al. 1987) are numbered as follows: (1) GX 1+4, (2) MXB 1730-335 ("Rapid Burster"), (3) SLX 1732-304, (4) SLX 1735-269, (5) SLX 1737-282, (6) A1743-322, (7) Sgr A* (8) A 1742-294, (9) 1E 1743.1-2843, (10) SLX $1744-299,(11)$ GX $3+1,(12)$ GRS $1758-258$, and (13) GX $5-1$.

energies from 35 to $200 \mathrm{keV}$ (Cook et al. 1990b). Details on the processing of coded-aperture images to obtain source flux measurements are given in Cook et al. (1984), Finger (1987), and Palmer et al. (1991).

Table 1 also includes results for three other sources: the Galactic nucleus radio source Sgr A*, the X-ray binary pulsar GX $1+4$, and the recently discovered $\gamma$-ray source GRS $1758-258$.

\section{DISCUSSION}

IE 1740.7-2942.-The identification of the strong, central $\gamma$-ray source (Fig. 1) as 1E 1740.7-2942 (Cook et al. 1990a) is

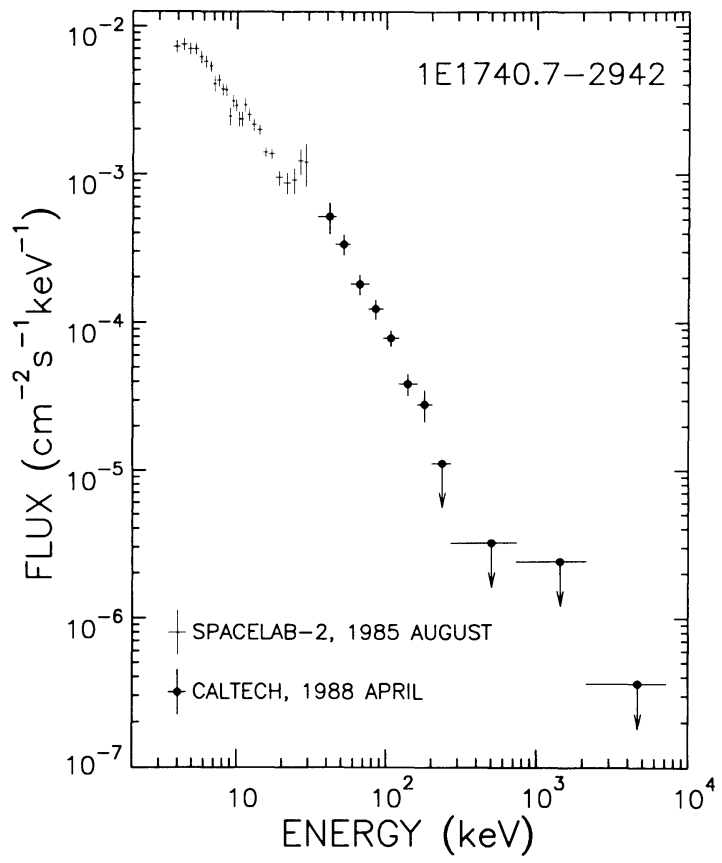

Fig. 2.-Gamma-ray differential energy spectrum measurements for the source 1E $1740.7-2942$. Error bars are $\pm 1 \sigma$, while upper limits are at the $95 \%$ confidence level.

consistent with the earlier imaging results from Spacelab 2 at energies below $30 \mathrm{keV}$ (Skinner et al. 1987). At energies above $30 \mathrm{keV}$, the recent imaging results from SIGMA confirm our identification of $1 \mathrm{E} 1740.7-2942$ as the primary hard X-ray source (Mandrou 1990). This source has not yet been identified at other than $\mathrm{X}$-ray and $\gamma$-ray wavelengths.

Our spectral results for 1E 1740.7-2942, and the earlier results from Spacelab 2 (Skinner et al. 1989) are shown together in Figure 2. The hard spectrum, which distinguished $1 \mathrm{E} 1740.7-2942$ from other nearby sources viewed by Spacelab 2 , is seen to continue to approximately $200 \mathrm{keV}$. In the energy range from 35 to $200 \mathrm{keV}$ the spectrum is well fit by a power law, $d J / d E=K(E / 100 \mathrm{keV})^{-\alpha}$, with spectral index

TABLE 1

Galactic Center Region Source Flux Values ${ }^{\mathrm{a}}$

\begin{tabular}{|c|c|c|c|c|c|c|}
\hline \multirow{2}{*}{$\begin{array}{l}\text { ENERGY } \\
\text { INTERVAL }\end{array}$} & \multicolumn{4}{|c|}{ DifFerential FluX $10^{-5}\left(\mathrm{~cm}^{2} \mathrm{~s} \mathrm{keV}\right)^{-1}$} & \multicolumn{2}{|c|}{ INTEGRAL FLUX $10^{-4}\left(\mathrm{~cm}^{2} \mathrm{~s}\right)^{-1}$} \\
\hline & $35-58 \mathrm{keV}$ & $58-122 \mathrm{keV}$ & $122-270 \mathrm{keV}$ & $270-490 \mathrm{keV}$ & $511 \mathrm{keV}^{\mathrm{b}}$ & $545-2150 \mathrm{keV}$ \\
\hline $\begin{array}{c}\text { 1E } 1740.7-2942 \\
(\alpha=2.05)\end{array}$ & $\begin{array}{c}40.6 \pm 5.5 \\
(47)\end{array}$ & $12.2 \pm 1.0$ & $\begin{array}{c}1.96 \pm 0.35 \\
(178)\end{array}$ & $\begin{array}{l}<0.47 \\
(378)\end{array}$ & $<6.8$ & $<46.1$ \\
\hline $\begin{array}{l}\mathrm{GX} 354+0 \\
(\alpha=3.37)\end{array}$ & $\begin{array}{c}38.6 \pm 10.8 \\
(46)\end{array}$ & $\begin{array}{c}6.12 \pm 2.03 \\
(79)\end{array}$ & $\begin{array}{l}<1.94 \\
(199)\end{array}$ & $\begin{array}{l}<0.92 \\
(378)\end{array}$ & $<7.1$ & $<18.0$ \\
\hline $\begin{array}{l}\mathrm{GX} 1+4 \\
(\alpha=1.9)\end{array}$ & $17.9 \pm 7.5$ & $\begin{array}{l}<4.1 \\
(93)\end{array}$ & $\begin{array}{l}<0.82 \\
(199)\end{array}$ & $\begin{array}{l}<0.69 \\
(378)\end{array}$ & $<3.7$ & $<27.0$ \\
\hline Sgr $A^{* c}$ & $\ldots$ & $\ldots$ & $\ldots$ & $\begin{array}{l}<0.65 \\
(378)\end{array}$ & $<11.7$ & $<52.1$ \\
\hline GRS $1758-258$ & $\begin{array}{l}<19.9 \\
(50)\end{array}$ & $\begin{array}{l}<3.1 \\
(93)\end{array}$ & $\begin{array}{l}<1.1 \\
(199)\end{array}$ & $\begin{array}{l}<0.89 \\
(378)\end{array}$ & $<7.8$ & $<22.8$ \\
\hline
\end{tabular}

a Differential flux values correspond to a power law $\left(d J / d E=K E^{-\alpha}\right)$ evaluated at the mean energy (keV), listed in parenthesis below the corresponding flux value. The exponent, $\alpha$, is given below the source name. Upper limits are quoted at $95 \%$ confidence, and assume a flat power law $(\alpha=0)$.

b The flux upper limits for a narrow line at $511 \mathrm{keV}$ were derived by dividing the integral flux limits for a $55 \mathrm{keV}$ wide energy interval by the fraction $(0.87)$ of $511 \mathrm{keV}$ photons which would be detected in that interval.

c Fluxes and upper limits are not given for the three lowest energy ranges. This is due to contamination from the nearby bright source $1 \mathrm{E} 1740.7-2942$. 
$\alpha=2.05 \pm 0.15$, and flux normalization, $K=8.5 \pm 0.5 \times 10^{-5}$ $\mathrm{cm}^{-2} \mathrm{~s}^{-1} \mathrm{keV}^{-1}$.

Our results for 1E 1740.7-2942 may also be compared to earlier nonimaging measurements. Our measured spectrum falls near the lower envelope of previous flux measurements for the Galactic center region obtained at energies from about 50 $\mathrm{keV}$ to $1 \mathrm{MeV}$ with nonimaging, wide aperture ( $\left.>10^{\circ} \mathrm{FWHM}\right)$ instruments (Matteson 1982). The higher fluxes obtained with these instruments at energies below $100 \mathrm{keV}$ are probably due to the inclusion of other (possibly time-variable) hard X-ray sources such as GX $354+0$ (see below) located within $10^{\circ}$ of the Galactic center. Several instruments (Dennis et al. 1980; Matteson 1982; Levine et al. 1984; Knight et al. 1985) with relatively narrow apertures ( 1.5 to $\left.5^{\circ} \mathrm{FWHM}\right)$ have observed a source (or sources) near the Galactic center with flux at 100 $\mathrm{keV}$ ranging from $0.5 \times 10^{-4}$ to $2.0 \times 10^{-4} \mathrm{~cm}^{-2} \mathrm{~s}^{-1} \mathrm{keV}^{-1}$, comparable to that shown in Figure 2. Our observations strongly support the suggestion by Skinner et al. (1987) that $1 \mathrm{E}$ $1740.7-2942$ was the source of the high energy flux seen in these earlier observations.

One possibility is that $1 \mathrm{E} 1740.7-2942$ is a system similar to the black hole candidate Cyg X-1 with several states of emission, one of which produces the spectrum shown in Figure 2, and another which yields hard $\mathrm{MeV}$ continuum and 0.511 $\mathrm{MeV}$ emission. Indeed, our measured spectrum for $1 \mathrm{E}$ $1740.7-2942$ is similar in absolute intensity and spectral shape to the $\gamma_{3}$ state of Cyg X-1, as measured by the $H E A O A-3$ experiment in 1979 (Ling et al. 1987). Our measurements for $1 \mathrm{E}$ $1740.7-2942$ yield a $35-200 \mathrm{keV}$ luminosity of $2.1 \times 10^{37} \mathrm{ergs}$ $\mathrm{s}^{-1}$ (at an assumed distance of $8.5 \mathrm{kpc}$ ), comparable to the $50-400 \mathrm{keV}$ luminosity measured for Cyg X-1 in its $\gamma_{3}$ state of $2.3 \times 10^{37}$ ergs s $^{-1}$ (Ling et al. 1987). Further, the spectrum of Cyg X-1 in its $\gamma_{1}$ state has similarities to the Galactic center spectrum measured in 1979, showing a hard excess of emission above $0.511 \mathrm{MeV}$ with comparable luminosity (Lingenfelter \& Ramaty 1989). Recently, a time variable hard shoulder of emission from 1E $1740.7-2942$, extending from $250-600 \mathrm{keV}$, has been reported by Mandrou et al. (1990).

$G X 354+0$.- The X-ray burster GX $354+0$ is a source of both persistent and transient X-ray emissions (e.g., Basinska et al. 1984; Hoffman et al. 1976; Hoffman et al. 1977). The X-ray intensity of the persistent source is known to vary by over a factor of $\sim 5$ (Basinska et al. 1984; Forman et al. 1978). The Einstein observatory measured the most intense and hot nonburst spectrum to date, and found it to be consistent with a thermal bremsstrahlung model having a temperature of $k T=17.7 \mathrm{keV}$ (Grindlay \& Hertz 1981).

Our flux measurements for GX $354+0$, averaged over the entire $8 \mathrm{hr}$ observation, are presented in Table 1. Comparison between our data and an extrapolation of the $17.7 \mathrm{keV}$ thermal bremsstrahlung spectrum, measured by the Einstein observatory, yields approximate agreement, suggesting that the source may have been near its maximum nonburst brightness during our observation. From our data, the best-fit temperature, with estimated $1 \sigma$ errors, is $k T=31(+23,-10) \mathrm{keV}$.

$G X 1+4$. - Observations of the X-ray binary pulsar GX $1+4$, in the decade following its discovery by Lewin, Ricker, \& McClintock (1971), showed the source to be one of the brightest hard X-ray sources in the Galactic center region, and to have an unusually large monotonic spin-up rate of approximately $2 \% \mathrm{yr}^{-1}$ (Elsner et al. 1985). In contrast, during the 1980 's the source was observed to be in a "low" state of emis- sion (Makishima et al. 1988; Manchanda 1988; Sakao et al. 1990) accompanied by a halt in the rapid spin up of the pulsar. Recent observations show the pulsar spinning down (Makishima et al. 1988; Gilfanov et al. 1989; Sunyaev 1990). McClintock \& Leventhal (1989) have proposed GX $1+4$ as a candidate for the compact source of Galactic center $0.511 \mathrm{MeV}$ emission, since this emission may have decreased in approximate correlation with the shift in observational properties of GX $1+4$.

While emission from GX $1+4$ is not apparent in the $23-122$ $\mathrm{keV}$ image of Figure 1, a greater than $2 \sigma$ flux measurement was obtained in the $35-58 \mathrm{keV}$ interval as listed in Table 1. This flux corresponds to $67 \pm 28 \mathrm{mCrab}$ at $50 \mathrm{keV}$ and is in agreement with the recent measurements of Mony et al. (1989) and Sharma et al. (1990).

GRS $1758-258$. - This source was recently discovered by the ART-P and SIGMA coded-aperture instruments aboard the GRANAT space observatory (Mandrou 1990). Our $95 \%$ confidence upper limit for the flux in the energy interval from 35 to $58 \mathrm{keV}$ corresponds to $78 \mathrm{mCrab}$, and is consistent with the ART-P result of $90 \pm 20 \mathrm{mCrab}$ for 20 to $40 \mathrm{keV}$.

$0.511 \mathrm{MeV}$ Line and $\mathrm{MeV}$ Continuum Upper Limits.-Our 1988 April imaging observations of the Galactic center have yielded no positive detection of either $0.511 \mathrm{MeV}$ line emission, or continuum emission at higher energies. Table 1 lists $95 \%$ confidence flux upper limits for the $0.511 \mathrm{MeV}$ line, and for a broad continuum band from 0.55 to $2.1 \mathrm{MeV}$, as obtained for 1E 1740.7-2942, Sgr A*, GX 1+4, GX 354+0, and GRS $1758-258$.

Sgr $A^{*}$, the compact Galactic nucleus radio source, and more recently $1 \mathrm{E} 1740.7-2942$ and GX $1+4$, have been suggested as candidates for the source of the variable Galactic center positron annihilation line and hard $\mathrm{MeV}$ continuum emission (Lingenfelter \& Ramaty 1982; Skinner et al. 1987; McClintock \& Leventhal 1989). This emission was seen to turn off or decrease between the HEAO 3 observations of 1979 Fall and 1980 Spring (Riegler et al. 1985). The flux decrease was most dramatic at energies near $1 \mathrm{MeV}$, where the continuum level dropped by a factor of 20 or more. The $0.511 \mathrm{MeV}$ radiation has recently been reported to have turned on again in 1988 (Leventhal et al. 1989; Gehrels et al. 1990), then decreased by 1989 May (Matteson et al. 1989).

Our $95 \%$ confidence flux upper limits for the $0.54-2.1 \mathrm{MeV}$ interval range from $1.8 \times 10^{-3}$ to $5.2 \times 10^{-3} \mathrm{~cm}^{-2} \mathrm{~s}^{-1}$, and are all well below the 1979 Fall HEAO 3 spectrum (Riegler et al. 1985), which, when integrated over the same energy range, gives $1.0 \pm 0.2 \times 10^{-2} \mathrm{~cm}^{-2} \mathrm{~s}^{-1}$.

Our $0 . \overline{5} 11 \mathrm{MeV}$ upper limits may be compared to recent Galactic center $0.511 \mathrm{MeV}$ line flux measurements made with nonimaging Ge spectrometers during 1988 May and October (Gehrels et al. 1991), and 1989 May (Matteson et al. 1989). These observations yielded values of $7.5 \pm 1.7,11.8 \pm 1.6$, and $6.5 \pm 1.9 \times 10^{-4} \mathrm{~cm}^{-2} \mathrm{~s}^{-1}$, respectively. Our $0.511 \mathrm{MeV}$ upper limits do not conflict with the 1988 May measurement (made only 18 days after our flight), since our limits apply for isolated points in our field of view and are relatively immune to the presence of diffuse flux. After adjustment for the diffuse $0.511 \mathrm{MeV}$ flux measured by $S M M$ (Share et al. 1988), the portion of the 1988 May measurement which might be attributed to a compact source is only $2.8 \pm 1.7 \times 10^{-4} \mathrm{~cm}^{-2} \mathrm{~s}^{-1}$ (Gehrels et al. 1990).

In summary, we see no evidence of $0.511 \mathrm{MeV}$ line emission 
from 1E 1740.7-2942, Sgr A*, GX 1+4, GX 354+0, or GRS $1758-258$. We also did not observe a continuum excess in the energy range $250-600 \mathrm{keV}$ like that reported by SIGMA (Mandrou et al. 1990) for 1E 1740.7-2942. However, the recent SIGMA observation provides evidence that transient positron annihilation may be important in 1E 1740.7-2942, strengthening the possibility that this was indeed the source of narrow positron annihilation radiation seen in observations during the 1970's and late 1980's.
We acknowledge the important contributions to the development of the GRIP telescope made by W. Althouse, D. Burke, A. Cummings, M. Finger, C. Starr, J. Weger, and the personnel of the Central Engineering Services at Caltech. We thank the personnel of the National Scientific Balloon Facility and the NASA Wallops Flight Facility for their excellent balloon launch support. This work was supported in part by NASA grants NGR 05-002-160 and NAGW-1919.
Althouse, W. E., Cook, W. R., Cummings, A. C., Finger, M. H., Prince, T. A., Schindler, S. M., Starr, C. H., \& Stone, E. C. 1985, Proc. 19th Internat. Cosmic Ray Conf. (La Jolla), 3, 299

Basinska, E. M., Lewin, W. H. G., Sztajno, M., Cominsky, L. R., \& Marshall, F. J. 1984, ApJ, 281, 337

Bradt, H. V., \& McClintock, J. E. 1983, ARA\&A, 21, 13

Cook, W. R., Finger, M., Prince, T. A., \& Stone, E. C. 1984, IEEE Trans. Nucl. Sci., NS-31, No. 1, 771

Cook, W. R., Grunsfeld, J. M., Heindl, W. A., Palmer, D. M., Prince, T. A., Schindler, S. M., Starr, C. H., \& Stone, E. C. 1990b, Adv. Space Res., in press Cook, W. R., Heindl, W. A., Palmer, D. M., Prince, T. A., Schindler, S. M., Starr, C. H., \& Stone, E. C. 1990a, Proc. 21st Internat. Cosmic Ray Conf. (Adelaide), 1,216

Cook, W. R., Palmer, D. M., Prince, T. A., Schindler, S. M., Starr, C. H., \& Stone, E. C. 1988, in IAU Symposium 136, The Center of the Galaxy, ed. M. Morris (Dordrecht: Reidel), p. 581

Dennis, B. R., Beall, J. H., Cutler, E. P., Crannell, C. J., Dolan, J. F., Frost, K. J., \& Orwig, L. E. 1980, ApJ, 236, L49

Elsner, R. F., Weisskopf, M. C., Apparao, K. M. V., Darbro, W., Ramsay, B. D., Williams, A. C., Grindlay, J. E., \& Sutherland, P. G. 1985, ApJ, 297, 288

Finger, M. 1987, Ph.D. thesis, California Institute of Technology

Forman, W., Jones, C., Cominsky, L., Julien, P., Murray, S., Peters, G., Tananbaum, H., \& Giacconi, R. 1978, ApJS, 38, 357

Gehrels, N., Barthelmy, S., Teegarden, B., Tueller, J. Leventhal, M., \& MacCallum, C. J. 1990, Bull. Am. Phys. Soc., 35, No. 4, 1081

- ApJ, submitted

Gilfanov, M., et al. 1989, in Proc. 23d ESLAB Symposium on Two-Topics in $\mathrm{X}$-Ray Astronomy, Bologna, Italy, 71

Grindlay, J. E., \& Hertz, P. 1981, ApJ, 247, L17

Hertz, P., \& Grindlay, J. E. 1984, ApJ, 278, 137

Hoffman, J. A., Lewin, W. H. G., \& Doty, J. 1977, MNRAS, 179, 57P

Hoffman, J. A., Lewin, W. H. G., Doty, J., Hearn, D. R., Clark, G. R. W., Jernigan, G., \& Li F. K. 1976, ApJ, 210, L13

Jung, G. V. 1989, ApJ, 338, 972

Kawai, N., Fenimore, E. E., Middleditch, J., Cruddace, R. G., Fritz, G. G., Snyder, W. A., \& Ulmer, M. P. 1988, ApJ, 330, 130

Knight, F. K., Johnson III, W. N., Kurfess, J. D., \& Strickman, M. S. 1985, ApJ, 290, 557

Leventhal, M., MacCallum, C. J., Barthelmy, S. D., Gehrels, N., Teegarden, B. J., \& Tueller, J. 1989, Nature, 339, 36
Leventhal, M., MacCallum, C. J., Huters, A. F., \& Stang, P. D. 1982, ApJ, 260, L1

Levine, A. M., et al. 1984, ApJS, 54, 581

Lewin, W. H. G., Ricker, G. R., \& McClintock, J. E. 1971, ApJ, 169, L17

Ling, J. C., Mahoney, W. A., Wheaton, W. A., \& Jacobson, A. S. 1987, ApJ, $321, \mathrm{~L} 117$

Lingenfelter, R. E., \& Ramaty, R. 1989, ApJ, 343, 686

. 1982, in AIP Conf. Proc. No. 83, The Galactic Center, ed. G. R. Riegler \& R. D. Blandford (New York: AIP), p. 148

Makishima, K., et al. 1988, Nature, 333, 746

Manchanda, R. K. 1988, A\&SS, 150, 31

Mandrou, P. 1990, IAU Circ., No. 5032

Mandrou, P., Roques, J. P., Sunyaev, R., Churazov, E., Paul, J., \& Cordier, B. 1990, IAU Circ. No. 5140

Matteson, J. L. 1982, in AIP Conf. Proc., No. 83, The Galactic Center, ed. C. R. Riegler \& R. D. Blandford (New York: AIP), p. 109

Matteson, J., et al. 1989, IAU Circ., No. 4889

McClintock, J. E., \& Leventhal, M. 1989, ApJ, 346, 143

Mony, B., et al. 1989, Proc. 23d ESLAB Symposium on Two-Topics in X-ray Astronomy, Bologna, Italy, 541

Palmer, D. M., Cook, W. R., Grunsfeld, J. M., Prince, T. A., Schindler, S. M., \& Stone, E. C. 1991, in preparation

Riegler, G. R., Ling, J. C., Mahoney, W. A., Wheaton, W. A., Willett, J. B., Jacobson, A. S., \& Prince, T. A. 1981, ApJ, 248, L13

Riegler, G. R., Ling, J. C., Mahoney, W. A., Wheaton, W. A., \& Jacobson, A. S. 1985, ApJ, 294, L13

Riegler, G. R., Ling, J. C., Mahoney, W. A., Wheaton, W. A., Willett, J. B., Jacobson, A. S., \& Prince, T. A. 1981, ApJ, 248, L13

Sakao, T., et al. 1990, MNRAS, 246, 11P

Skinner, G. K., et al. 1987, Nature, 330, 544

Skinner, G. K., Willmore, A. P., Foster, A. J., \& Eyles, C. J. 1989, Proc. Gamma Ray Observatory Science Workshop, ed. N. Johnson, (Greenbelt: GSFC) 4-191

Share, G. H., Kinzer, R. L., Kurfess, J. D., Messina, D. C., Purcell, W. R., Chupp, E. L., Forrest, D. J., \& Reppin, C. 1988, ApJ, 327, 717

Sharma, D. P., et al. 1990, Proc. 21st Internat. Cosmic Ray Conf. (Adelaide), 1, 32

Sunyaev, R., \& the GRANAT team 1990, IAU Circ., No. 5104

Watson, M. G., Willingale, R., Grindlay, J. E., \& Hertz, P. 1981, ApJ, 250, 142

Note added in proof.-A recent report by Paul et al. (in Proc. Internat Symp. on Gamma-Ray Line Astrophysics, Paris-Saclay, in press [1991]), based on SIGMA observations of the 1990 October 13 high-energy flare (250-600 keV) from 1E1740.7-2942 (Mandrou et al. 1990), strengthens the interpretation of the event as being associated with an electron-positron pair plasma. 